\title{
COUNTS-AS: CLASSIFICATION OR CONSTITUTION? AN ANSWER USING MODAL LOGIC
}

\author{
Davide Grossi, John-Jules Ch. Meyer, Frank Dignum \\ Utrecht University, \\ The Netherlands \\ \{davide,jj,dignum\}@cs.uu.nl
}

\begin{abstract}
By making use of modal logic techniques, the paper disentangles two semantically different readings of statements of the type $X$ counts as $Y$ in context $C$ (the classificatory and the constitutive readings) showing that, in fact, 'counts-as is said in many ways'.
\end{abstract}

\section{Introduction}

The term "counts-as" derives from the paradigmatic formulation that in [19] and [20] is attributed to the non-regulative component of institutions, i.e., constitutive rules:

[...] "institutions" are systems of constitutive rules. Every institutional fact is underlain by a (system of) rule(s) of the form " $\mathrm{X}$ counts as $\mathrm{Y}$ in context C" ( [19], pp.51-52).

In legal theory the non-regulative component of normative systems has been labeled in ways that emphasize a classificatory, as opposed to a normative or regulative, character: conceptual rules ( [2]), qualification norms ( [18]), definitional norms ( [13]). Constitutive rules are definitional in character:

The rules for checkmate or touchdown must 'define' checkmate in chess or touchdown in American Football [...] ( [19], p.43).

With respect to this feature, a first reading of counts-as is thus readily available: it is plain that counts-as statements express classifications. For example, they express what is classified to be a checkmate in chess, or a touchdown in American Football. However, is this all that is involved in the meaning of counts-as statements?

The interpretation of counts-as in merely classificatory terms does not do justice to the notion which is stressed in the label "constitutive rule", that is, the notion of constitution. Aim of the paper is to show that this notion, as it is presented in some work in legal and social theory, is amenable to formal characterization (in modal logic) and that the theory we developed in [11] provides a ground for its understanding. These investigations stem therefore from the acknowledgment that what is commonly studied under the label "counts-as", hides in fact (at least) two different, though related, phenomena. Aim of the work is to 
disentangle these two different meanings and to do it formally. The result will be a formal characterization of both of them together with their logical relations.

The work is structured as follows. In Section 2 we provide an informal analysis of the differences between the classificatory and the constitutive readings and we isolate some constraints concerning in particular the semantics of the constitutive reading. In Section 3 we expose a modal logic of contextual classifications and by means of it we make explicit what a classificatory view of counts-as implies. In Sections 4 and Section 5 a formal characterization of the constitutive view is instead provided and some logical interdependencies between the two readings are derived. Section 6 is devoted to a brief discussion of what we deem to be the most characteristic features of our approach with respect to the literature available on the topic. Conclusions follow in Section 7.

\section{Counts-as between Classification and Constitution}

Consider the following inference: it is a rule of normative system $\Gamma$ that selfpropelled conveyances count as vehicles; it is always the case that cars count as self-propelled conveyances; therefore, according to normative system $\Gamma$, cars count as vehicles. This is an instance of a typical normative reasoning pattern: from the rule of a given normative system and a common-sense fact, another fact is inferred which holds with respect to that normative system. The count-as locution occurs three times. However, the first premise states a constitutive rule, the second one states a generally acknowledged classification, and the conclusion states a classification which is considered to hold with respect to the given normative system. The formal analysis proposed in this paper moves from the observation that these different occurrences of the same term counts-as denote, in effect, different concepts. Counts-as can be said in many ways, and the aim of the paper is to show that these 'many ways' all have a precise formal semantics.

The distinction we are going to focus on can be distilled in the following observation: counts-as statements used to express constitutive rules have a different meaning from the counts-as statements which are instead used for expressing what follows from the existence of a constitutive rule. We call the first ones constitutive counts-as statements, and the second ones classificatory counts-as statements. We will see (Proposition 5) that the formal counterpart of the above reasoning pattern is a validity of the modal logic framework that we are going to introduce for capturing and analyzing this distinction.

\subsection{The classificatory reading of counts-as}

According to a classificatory perspective on the semantics of counts-as, such as the one we investigated in [9-11], the fact that $A$ counts-as $B$ in context $c$ is interpreted as " $A$ is a subconcept of $B$ in context $c$ ". In other words, counts-as statements are read as contextual classifications.

A notion of context is necessary because classifications holding for a normative system are not of a universal kind, they do not hold in general. What does 
this mean? The set of classifications stated as constitutive rules by a normative system (for instance, "self-propelled conveyances count as vehicles") can be seen as exactly the set of situations (possible worlds) which make that set of classifications true. Hence, the set of constitutive rules of any normative system can be seen as a set of situations. And a set of situations is what is called a context in much literature on context theory (see for instance $[7,22]$ ). To put it in a nutshell, a context is a set of situations, and if the constitutive rules of a given normative system $\Gamma$ are satisfied by all and only the situations in a given set, then that set of situations is the context defined by $\Gamma^{1}$. This simple observation allows us to think of contexts as "systems of constitutive rules" ( [19], p.51). Getting back to the above example: the statement "according to $\Gamma$, cars count as vehicles" is read as "in the set of situations defined by the rules of system $\Gamma$, car is a subconcept of vechicle". These features, here just informally introduced, will be fully captured by the formalism we are going to present in the following sections.

Understanding counts-as statements in classificatory terms sheds light on an essential function of constitutive rules within normative systems, namely the function of specifying the ontology that each normative system presupposes in order to be able to carry out its regulative task ( [8]). Counts-as statements describe the ontology that normative systems use in order to distribute obligations, rights, prohibitions, permissions: vehicles are not admitted to public parks (general norm), but then, if cars count as vehicles (classification), cars are not admitted to public parks (specific norm). An analysis in terms of contextual classification captures exactly this type of reasoning patterns enabled by counts-as statements.

\subsection{The constitutive reading of counts-as}

The classificatory perspective does not exhaust, though, all aspects involved in the meaning of counts-as statements. As the analysis developed in [14] shows, there is something more. According to that work, the fact that $A$ counts-as $B$ in context $c$ is read as a statement to the effect that $A$ represents "conditions for guaranteeing the applicability of particular classificatory categories" [14], in this case the category $B$ in context $c$. One is not generally entitled to infer $B$ from $A$, but it is the counts-as itself which guarantees the soundness of that inference. Indeed, if we can say that cars count as vehicles in $c$, is just because there is a constitutive rule of normative system $\Gamma$ defining $c$, which states that self-propelled conveyances are vehicles. Without this constitutive rule the conclusion could not be drawn. As said in [14], the constitutive rule "guarantees" the soundness of the inference. The constitutive reading of counts-as stresses exactly this aspect.

\footnotetext{
1 The definition of sets of situations via sets of formulae is a well-known formal phenomenon, which has been object of deep investigations especially in epistemic logic. We will come back to this in more detail in Section 4 and 5.
} 
In this view, counts-as statements do not only state contextual classifications, but they state new classifications which would not otherwise hold. This is perfectly in line with what maintained in [19]:

[...] where the rule (or systems of rules) is constitutive, behaviour which is in accordance with the rule can receive specifications or descriptions which it could not receive if the rules did not exist [p.35] ( [19]).

In other words, $A$ counts as $B$ in context $c$ because, in general, it does not hold that $A$ is classified as $B$. Otherwise such a constitutive rule would be futile.

Remark 1. Constitutive counts-as statements are classifications which hold with respect to a context (set of situations) but which do not hold in general (i.e., with respect to all situations).

There is yet another feature characterizing the constitutive reading of countsas. Let us go back to the first premise of our example: it is a rule of normative system $\Gamma$ that self-propelled conveyances count as vehicles. Being normative systems sets of rules, this means that "self-propelled conveyances are classified as vehicles" is one of the rules specifying $\Gamma$. We know that any set of rules defines a context, namely, the context of all and only the situations which satisfy that set of rules, so:

Remark 2. A constitutive counts-as statement is a classification such that: a) it is an element of the set of rules specifying a given normative system $\Gamma$; $b)$ the set of rules of $\Gamma$ define the context (set of situations) to which the counts-as statement pertains.

In other words when statements " $A$ counts as $B$ in the context $c$ of normative system $\Gamma$ " are read as constitutive rules, what is meant is that the classification of $A$ under $B$ is a promulgation of the normative system $\Gamma$ defining context $c$.

Finally, let us spend some preliminary words about the relation between the classificatory and the constitutive reading which we should expect to be enabled in a framework capturing both those meanings. As the informal analysis above points out, the classificatory view is somehow implied by the constitutive one: a constitutive counts-as does not only express that $A$ is classified as $B$ in $c$, but it expresses also that this classification is not a universally valid one, and that it is an element of the the system $\Gamma$ defining $c$. A clear logical relation between the two views should therefore be expected.

Remark 3. Constitution implies classification: if $A$ counts as $B$ in a constitutive sense, then $A$ counts as $B$ in a classificatory sense.

Such a logical relation is precisely the ground for the type of reasoning typically involved in the manipulation of constitutive rules. The presence of a constitutive rule entitles the reasoner to apply reasoning patterns which are typical of reasoning with concepts and classifications. This aspect is thoroughly investigated in Section 5.5.

Along the lines just sketched, the work presents a proposal for developing the formal analysis we presented in [11] in order to deliver a unified modal logic 
framework able to capture both the constitutive and the classificatory views of counts-as.

\section{Modal logic of Classificatory Counts-as}

This section summarizes the results presented in [11]. We first introduce the languages we are going to work with: propositional n-modal languages $\mathcal{M} \mathcal{L}_{n}$ ( [5]). The alphabet of $\mathcal{M} \mathcal{L}_{n}$ contains: a finite set $\mathbb{P}$ of propositional atoms $p$; the set of boolean connectives $\{\neg, \wedge, \vee, \rightarrow\}$; a finite non-empty set of $n$ (context) indexes $C$, and the operator [ ]. Metavariables $i, j, \ldots$ are used for denoting elements of $C$. The set of well formed formulas $\phi$ of $\mathcal{M} \mathcal{L}_{n}$ is then defined by the following BNF:

$$
\phi::=\perp|p| \neg \phi\left|\phi_{1} \wedge \phi_{2}\right| \phi_{1} \vee \phi_{2}\left|\phi_{1} \rightarrow \phi_{2}\right|[i] \phi .
$$

We will refer to formulae $\phi$ in which at least one modal operator occurs as modalized formulae. We call instead objective formulae in which no modal operator occur and we denote them using the metavariables $\gamma_{1}, \gamma_{2}, \ldots$.

\subsection{Semantics}

Semantics for these languages is given via structures $\mathcal{M}=\langle\mathcal{F}, \mathcal{I}\rangle$, where:

- $\mathcal{F}$ is a CXT multi-frame, i.e., a structure $\mathcal{F}=\left\langle W,\left\{W_{i}\right\}_{i \in C}\right\rangle$, where $W$ is a finite set of states (possible worlds) and $\left\{W_{i}\right\}_{i \in C}$ is a family of subsets of $W$.

- $\mathcal{I}$ is an evaluation function $\mathcal{I}: \mathbb{P} \longrightarrow \mathcal{P}(W)$ associating to each atom the set of states which make it true.

Some observations are in order here. To put it another way, CxT multi-frames can be seen as Kripke frames in which, instead of the family of sets $\left\{W_{i}\right\}_{i \in C}$, a family of accessibility relations $\left\{R_{i}\right\}_{i \in C}$ is given which defines for each world $w$ the same set of accessible worlds $W_{i}$. Relations enjoying such a property are called locally universal ${ }^{2}$. Such multi-frames model thus $\mathrm{n}$ different contexts $i$ which might be inconsistent, if the corresponding set $W_{i}$ is empty, or global if $W_{i}$ coincides with $W$ itself. This implements in a straightforward way the thesis developed in context modeling according to which contexts can be soundly represented as sets of possible worlds ( [22]).

Satisfaction for modal formulae of these languages is then defined as follows:

$$
\mathcal{M}, w \vDash[i] \phi \text { iff } \forall w^{\prime} \in W_{i}: \mathcal{M}, w^{\prime} \vDash \phi .
$$

Satisfaction of atoms and boolean formulae is omitted and runs as usual. A formula $\phi$ is said to be valid in a model $\mathcal{M}$, in symbols $\mathcal{M} \vDash \phi$, iff for all $w$ in $W, \mathcal{M}, w \vDash \phi$. It is said to be valid in a frame $\mathcal{F}(\mathcal{F} \vDash \phi)$ if it is valid in all

\footnotetext{
${ }^{2}$ See [11] for a detailed discussion of these frames.
} 
models based on that frame. Finally, it is said to be valid on a class of frames $\mathrm{F}$ $(\mathrm{F} \vDash \phi)$ if it is valid in every frame $\mathcal{F}$ in $\mathrm{F}$.

It is instructive to make a remark about the $[i]$-operator clause, which can be seen as the characterizing feature of the modeling of contexts as sets of worlds ${ }^{3}$. It states that the truth of a modalized formula abstracts from the point of evaluation of the formula. In other words, the notion of "truth in a context $i$ " is a global notion: $[i]$-formulae are either true in every state in the model or in none. This reflects the idea that what is true or false in a context does not depend on the world of evaluation, and this is what we would intuitively expect especially for contexts interpreted as normative systems: what holds in the context of a given normative system is not determined by the point of evaluation but just by the system in itself, i.e., by its rules.

\subsection{Axiomatics}

The multi-modal logic that corresponds, i.e., that is sound and complete with respect to the class of CXT multi-frames, is a system we call here $\mathbf{K} \mathbf{4 5} \mathbf{i j}_{\mathbf{n}}^{\mathbf{i j}}$. It consists of a logic weaker than the logic $\mathbf{K D} 4 \mathbf{5}_{\mathbf{n}}^{\mathbf{i j}}$ investigated in [11] in that the semantic constraint has been dropped which required the sets in family $\left\{W_{i}\right\}_{i \in C}$ to be non-empty. As a consequence the $\mathrm{D}$ axiom is eliminated. To put it in a nutshell, the system is the very same logic for contextual classification developed in [11] except for the fact the we want to allow here the representation of empty contexts as well. In the knowledge representation setting we are working in, where contexts can be identified with the normative systems defining them, this amounts to accept the possibility of normative systems issuing inconsistent constitutive rules.

Logic $\mathbf{K} 45_{\mathbf{n}}^{\mathbf{i j}}$ is axiomatized via the following axioms and rules schemata:

(P) all tautologies of propositional calculus

$(\mathrm{K})[i]\left(\phi_{1} \rightarrow \phi_{2}\right) \rightarrow\left([i] \phi_{1} \rightarrow[i] \phi_{2}\right)$

$\left(4^{i j}\right)[i] \phi \rightarrow[j][i] \phi$

$\left(5^{i j}\right) \neg[i] \phi \rightarrow[j] \neg[i] \phi$

(MP) $\phi_{1}, \phi_{1} \rightarrow \phi_{2} / \phi_{2}$

(N) $\phi /[i] \phi$

where $i, j$ denote elements of the set of indexes $C$. The system is a multi-modal homogeneous $\mathbf{K} 45$ with the two interaction axioms $4^{i j}$ and $5^{i j}$. The system is a subsystem of the EDL system studied in [17]. The proof of the soundness and completeness of the system with respect to CXT multi-frames can be derived by the proof of the completeness of EDL ( [17]).

A remark is in order especially with respect to axiomata $4^{i j}$ and $5^{i j}$. In fact, what the two schemata do, consists in making the nesting of the operators reducible which, leaving technicalities aside, means that truth and falsehood in

\footnotetext{
${ }^{3}$ Propositional logics of context without this clause are investigated in $[3,4]$.
} 
contexts $([i] \phi$ and $\neg[i] \phi)$ are somehow absolute because they remain invariant even if evaluated from another context $([j][i] \phi$ and $[j] \neg[i] \phi)$. In other words, they express the fact that whether something holds in a context $i$ is not something that a context $j$ can influence. This is indeed the kind of property to be expected given the semantics presented in the previous section.

\subsection{Classificatory Counts-as formalized}

Using a multi-modal logic $\mathbf{K} 45_{\mathbf{n}}^{\mathrm{ij}}$ on a language $\mathcal{M} \mathcal{L}_{n}$, the formal characterization of the classificatory view on counts-as statements runs as follows.

Definition 1. (Classificatory counts-as: $\Rightarrow_{c}^{c l}$ )

" $\gamma_{1}$ counts as $\gamma_{2}$ in context $c$ " is formalized in a multi-modal language $\mathcal{M} \mathcal{L}_{n}$ as the strict implication between two objective sentences $\gamma_{1}$ and $\gamma_{2}$ in logic $\mathbf{K} \mathbf{4 5} \mathbf{n}_{\mathbf{n}}^{\mathrm{ij}}$ :

$$
\gamma_{1} \Rightarrow_{c}^{c l} \gamma_{2}:=[c]\left(\gamma_{1} \rightarrow \gamma_{2}\right)
$$

These properties for $\Rightarrow_{c}^{c l}$ follow.

Proposition 1. (Properties of $\Rightarrow_{c}^{c l}$ )

In logic $\mathbf{K} 45_{\mathbf{n}}^{\mathbf{i j}}$, the following formulas and rules are valid:

$$
\begin{aligned}
& \gamma_{2} \leftrightarrow \gamma_{3} /\left(\gamma_{1} \Rightarrow_{c}^{c l} \gamma_{2}\right) \leftrightarrow\left(\gamma_{1} \Rightarrow_{c}^{c l} \gamma_{3}\right) \\
& \gamma_{1} \leftrightarrow \gamma_{3} /\left(\gamma_{1} \Rightarrow_{c}^{c l} \gamma_{2}\right) \leftrightarrow\left(\gamma_{3} \Rightarrow_{c}^{c l} \gamma_{2}\right) \\
& \left(\left(\gamma_{1} \Rightarrow_{c}^{c l} \gamma_{2}\right) \wedge\left(\gamma_{1} \Rightarrow_{c}^{c l} \gamma_{3}\right)\right) \rightarrow\left(\gamma_{1} \Rightarrow_{c}^{c l}\left(\gamma_{2} \wedge \gamma_{3}\right)\right) \\
& \left(\left(\gamma_{1} \Rightarrow_{c}^{c l} \gamma_{2}\right) \wedge\left(\gamma_{3} \Rightarrow_{c}^{c l} \gamma_{2}\right)\right) \rightarrow\left(\left(\gamma_{1} \vee \gamma_{3}\right) \Rightarrow_{c}^{c l} \gamma_{2}\right) \\
& \gamma \Rightarrow_{c}^{c l} \gamma \\
& \left(\gamma_{1} \Rightarrow_{c}^{c l} \gamma_{2}\right) \wedge\left(\gamma_{2} \Rightarrow_{c}^{c l} \gamma_{3}\right) \rightarrow\left(\gamma_{1} \Rightarrow_{c}^{c l} \gamma_{3}\right) \\
& \left(\gamma_{1} \Rightarrow_{c}^{c l} \gamma_{2}\right) \wedge\left(\gamma_{2} \Rightarrow_{c}^{c l} \gamma_{1}\right) \rightarrow[c]\left(\gamma_{1} \leftrightarrow \gamma_{2}\right) \\
& \left(\gamma_{1} \Rightarrow_{c}^{c l} \gamma_{2}\right) \rightarrow\left(\gamma_{1} \wedge \gamma_{3} \Rightarrow_{c}^{c l} \gamma_{2}\right) \\
& \left(\gamma_{1} \Rightarrow_{c}^{c l} \gamma_{2}\right) \rightarrow\left(\gamma_{1} \Rightarrow_{c}^{c l} \gamma_{2} \vee \gamma_{3}\right)
\end{aligned}
$$

We omit the proofs, which are straightforward via application of Definition 1. This system validates all the intuitive syntactic constraints isolated in [14] (validities 1-5). In addition, this semantic-oriented approach to classificatory countsas enables the four validities 6-9. Besides, this analysis shows that counts-as conditionals, once they are viewed as conditionals of a classificatory nature, naturally satisfy reflexivity (5), transitivity (6), and a form of "contextualized" antisymmetry (7), strengthening of the antecedent (8) and weakening of the consequent (9).

The property of transitivity, in particular, deserves a special comment. In [14] the transitivity of counts-as is accepted, but not with strong conviction: "we have been unable to produce any convincing counter-instances and are inclined to accept it" ( $[14]$, p.436). What our approach shows is that once we first proceed to the isolation of the exact meaning we are aiming at formalizing, no room for uncertainty is then left about the syntactic properties enjoyed by the formalized notion: if we intend counts-as statements as contextual classifications, then transitivity must be accepted on the ground of pure logical reasons. 


\section{Counts-as Beyond Contextual Classification}

The previous section has provided a formal analysis of the classificatory view of counts-as (Definition 1), explicating what logical properties are to be expected once such an analytical option on the semantics of counts-as is assumed (Proposition 1). In this section, on the basis of Remark 1 and 2, we develop a formal semantics of the constitutive reading of counts-as.

\subsection{From Classification to Constitution}

What has to be done is just to give formal clothes to Remarks 1 and 2 stated in Section 2. Let us define the set $\mathbb{T}(X)$ of all formulae which are satisfied by all worlds in a finite set of worlds $X$ in a $\mathrm{CXT}$ model $\mathcal{M}$ :

$$
\mathbb{T}(X)=\{\phi \mid \forall w \in X: \mathcal{M}, w \models \phi\} .
$$

and the set $\mathbb{T} \rightarrow(X)$ of all implications between objective formulae which are satisfied by all worlds in a finite set of worlds $X$ :

$$
\mathbb{T}^{\rightarrow}(X)=\left\{\gamma_{1} \rightarrow \gamma_{2}|\forall w \in X: \mathcal{M}, w|=\gamma_{1} \rightarrow \gamma_{2}\right\}
$$

Obviously, for every $X: \mathbb{T}^{\rightarrow}(X) \subseteq \mathbb{T}(X)$. In the classificatory reading, given a model $\mathcal{M}$ where the set of worlds $W_{c} \subseteq W$ models context $c$, the set of all classificatory counts-as statements holding in $c$, i.e., $\mathbb{C} \mathbb{L}\left(W_{c}\right)$, is nothing but the set $\mathbb{T} \rightarrow\left(W_{c}\right)$ :

$$
\mathbb{C} \mathbb{L}\left(W_{c}\right) \equiv \mathbb{T}^{\rightarrow}\left(W_{c}\right) .
$$

Obviously, $\mathbb{C} \mathbb{L}\left(W_{c}\right)$ is a superset of all conditional truths of $W$, that is, of the "universal" context of model $\mathcal{M}$. This is to say that interpreting counts-as as a mere classification makes it inherit all trivial classifications which hold globally in the model, and in this consists precisely the crux of the failure of contextual classifications in capturing a notion of constitution. The notion of contextual classification is indifferent to what the context adds to standard classifications.

This suggests, though, a readily available strategy to give a formal specification of Remark 1: the set of constitutive counts-as statements holding in a context $c$ should be contained in the set $\mathbb{T} \rightarrow\left(W_{c}\right)$ from which all the global classifications are eliminated:

$$
\mathbb{C O}\left(W_{c}\right) \subseteq \mathbb{T}^{\rightarrow}\left(W_{c}\right) \backslash \mathbb{T}(W)
$$

Intuitively, the set of constitutive counts-as holding in $c$ corresponds to the set of implications holding in $c$, i.e. the set of classificatory counts-as statements of $c$, minus those classifications which hold globally.

As to Remark 2, what comes to play a role is the notion of a definition of the context of a counts-as statement. A definition of a context $c$ is a set of objective formulae $\Gamma$ such that, $\forall w \in W$ :

$$
\mathcal{M}, w \models \Gamma \text { iff } w \in W_{c} .
$$


that is, the set of forumlae $\Gamma$ such that all and only the worlds in $W_{c}$ satisfy $\Gamma$.

In practice, we are making use, in a different setting but with exactly analogous purposes, of a well-known technique developed in the modal logic of knowledge, i.e., the interpretation of modal operators on "inaccessible worlds" typical, for instance, of the "all that I know" epistemic logics ( [15]). Consequently, when it comes to Remark 2 we do not just refer to a context, but also (even if often implicitly) to the set of formulae (or rules) that define it. From a formal point of view constitutive counts-as should thus be indexed not only by the context they pertain to, but also by the definition of the context:

$$
\mathbb{C O}_{\Gamma}\left(W_{c}\right) \subseteq\left\{\gamma_{1} \rightarrow \gamma_{2} \mid \gamma_{1} \rightarrow \gamma_{2} \in \Gamma \text { and } \forall w\left(\mathcal{M}, w \models \Gamma \text { iff } w \in W_{c}\right)\right\}
$$

That is to say, the set of constitutive counts-as statements of a context $c$ w.r.t. a definition $\Gamma$ should be a subset of the set of implications which belong to the set of formulae $\Gamma$ defining $c$.

If we take the constraints expressed in formulae 10 and 11 to exhaust the meaning of a constitutive view of counts-as, then the set of constitutive counts-as statements of a given set $c$ w.r.t. a definition $\Gamma$ can be defined as follows.

Definition 2. (Set of constitutive counts-as in $c$ w.r.t. definition $\Gamma$ ) The set $\mathbb{C O}_{\Gamma}\left(W_{c}\right)$ of constitutive counts-as statements of a context $c$ defined by $\Gamma$ is:

$$
\mathbb{C O}_{\Gamma}\left(W_{c}\right):= \begin{cases}\left(\mathbb{\mathbb { T }} \rightarrow\left(W_{c}\right) \backslash \mathbb{T}(W)\right) \cap \Gamma, \text { if: } \forall w\left(\mathcal{M}, w \models \Gamma \text { iff } w \in W_{c}\right) \\ \emptyset, \quad \text { otherwise. }\end{cases}
$$

Section 5 is devoted to the development of a modal logic based on this definition and to a detailed analysis of this interpretation of counts-as statements especially in relation with the analytical option of viewing counts-as as mere contextual classificatory statements.

\section{Counts-as as Constitution}

In the following section a logic is developed which implements the above definition 2. By doing this, all the following requirements will be met at the same time: first, capture the intuitions discussed in Section 2 concerning the intuitive reading of counts-as statements in constitutive terms (Remark 1 and 2); second, maintain the possible worlds semantics of context exposed in Section 3 and developed in order to account for the classificatory view of counts-as; third, account for the logical relation between classificatory and constitutive counts-as (Remark 3).

\subsection{Expanding $\mathcal{M} \mathcal{L}_{n}$}

Language $\mathcal{M L}_{n}$ is expanded as follows. The set of context indexes $C$ consists of a finite non-empty set $K$ of $m$ atomic indexes $c$ among which the special context 
index $u$ denoting the universal context, and their negations $-c$. The following morphological clause is thus needed:

$$
k::=c \mid-c
$$

The cardinality $n$ of $K$ is obviously equal to $2 m$. We call this language $\mathcal{M} \mathcal{L}_{n}^{u,-}$. Metavariables $i, j, \ldots$ for context identifiers range on the elements of $C$.

\subsection{Semantics}

Languages $\mathcal{M} \mathcal{L}_{n}^{u,-}$ are given a semantics via a special class of CxT multi-frames, namely the class of CxT multi-frames $\mathcal{F}=\left\langle W,\left\{W_{c}\right\}_{c \in C}\right\rangle$ such that there always exists a $W_{u} \in\left\{W_{c}\right\}_{c \in C}$ s.t. $W_{u}=W$. That is, the frames in this class, which we call $\mathrm{CxT}^{\top}$, always contain the global context among their contexts.

The semantics for $\mathcal{M L}_{n}^{u,-}$ is thus easily obtained interpreting the formulae on models built on $\mathrm{CxT}^{\top}$ frames. The new clauses needed to be added to the definition of the satisfaction relation in Section 3 are the following ones:

$$
\begin{aligned}
& \mathcal{M}, w \vDash[u] \phi \text { iff } \forall w^{\prime} \in W_{u}: \mathcal{M}, w^{\prime} \vDash \phi \\
& \mathcal{M}, w \vDash[-c] \phi \text { iff } \forall w^{\prime} \in W \backslash W_{c}: \mathcal{M}, w^{\prime} \vDash \phi .
\end{aligned}
$$

Intuitively, the first clause states that the $[u]$ operator is interpreted on the universal 1-frame contained in each $\mathrm{CxT}^{\top}$ multi-frame, and the second states that the $[-c]$ operator ranges over the complement of the set $W_{c}$ on which $[c]$ instead ranges.

In fact, the $[c]$ operator specifies a lower bound on what holds in context $c$ ('something more may hold in $i$ '), that is, a formula $[c] \phi$ means that $\phi$ at least holds in context $c$. The $[-c]$ operator, instead, specifies an upper bound on what holds in $c$ ('nothing more holds in $c^{\prime}$ ), and a $[-c] \phi$ formula means therefore that $\phi$ at most does hold in $c$, i.e., $\phi$ at least does hold in the complementary context of $c^{4}$.

\subsection{Axiomatics}

To axiomatize the above semantics an extension of logic $\mathbf{K} 4 \mathbf{5}_{\mathbf{n}}^{\mathbf{i j}}$ is needed which can characterize also atomic context complementation. The extension, which we call logic $\mathbf{C x t}^{\mathbf{u}}, \backslash$, results from the union $\mathbf{K} \mathbf{4} 5_{\mathbf{n}}^{\mathbf{i j}} \cup \mathbf{S} \boldsymbol{5}_{\mathbf{u}}$, that is, from the union of $\mathbf{K} \mathbf{4 5} \mathbf{\mathbf { i j }}$ with the $\mathbf{S} \mathbf{5}_{\mathbf{u}}$ logic for the $[u]$ operator together with the interaction axioms $(\subseteq . u i)(\subseteq . u c)$ and $(-. \neg)$ below. The axiomatics is thus as follows:

(P) all tautologies of propositional calculus

$\left(\mathrm{K}^{i}\right)[i]\left(\phi_{1} \rightarrow \phi_{2}\right) \rightarrow\left([i] \phi_{1} \rightarrow[i] \phi_{2}\right)$

$\left(4^{i j}\right)[i] \phi \rightarrow[j][i] \phi$

$\left(5^{i j}\right) \neg[i] \phi \rightarrow[j] \neg[i] \phi$

$\left(\mathrm{T}^{u}\right)[u] \phi \rightarrow \phi$

\footnotetext{
${ }^{4}$ For an extensive discussion of this technique we refer the reader to [17].
} 


$$
\begin{aligned}
(\subseteq . u i) & {[u] \phi \rightarrow[i] \phi } \\
(\subseteq . u c) & {[c] \phi \wedge[-c] \phi \rightarrow[u] \phi } \\
(-. \neg) & {[-c] v \rightarrow \neg[c] v } \\
(\mathrm{MP}) & \phi_{1}, \phi_{1} \rightarrow \phi_{2} / \phi_{2} \\
\left(\mathrm{~N}^{i}\right) & \phi /[i] \phi
\end{aligned}
$$

where $i, j$ are metavariables for the elements of $K, c$ denotes elements of the set of atomic context indexes $C$, and $v$ ranges over uniquely satisfiable objective formulae, i.e., "objective" formulae which are true in at most one world.

The interaction axioms deserve some comments. Axiom $(\subseteq . u i)$ is quite intuitive. It just says that what holds in the global context, holds in every context. Axiom $(\subseteq . u c)$ is needed in order to axiomatize the interplay between atomic contexts and their complements: if some formula holds in both a context and its complement, than it holds globally. Axiom $(-. \neg)$ states instead that the contexts denoted by $c$ and $-c$ are strongly disjoint, in the sense that they do not contain the same valuations.

The system is nothing but a multi-modal version of a fragment of the $\mathbf{S 5 O}$ system investigated in [17] (that system contained also a "strongly universal context", that is the context of all logically possible valuations), which is in turn an extension of the propositional fragment of the "all I know" logic studied in [15]. We conjecture that the completeness proof can be obtained extending the completeness result concerning $\mathbf{S 5 O}$ provided in [17].

\subsection{Constitutive Counts-as formalized}

Using a multi-modal logic $\mathbf{C x t}{ }^{\mathbf{u}, \backslash}$ on a language $\mathcal{M L}_{n}^{u,-}$, the constitutive reading of counts-as statements can be formalized as follows.

Definition 3. (Constitutive counts-as: $\Rightarrow_{c, \Gamma}^{c o}$ )

Given a set of formulae $\Gamma$ the conjunction on which is $\Gamma=\left(\phi_{1} \wedge \ldots \wedge\left(\gamma_{1} \rightarrow\right.\right.$ $\left.\gamma_{2}\right) \wedge \ldots \wedge \phi_{n}$ ), the constitutive counts-as statement " $\gamma_{1}$ counts as $\gamma_{2}$ in context $c "$ is formalized in a multi-modal logic $\mathbf{C x t}^{\mathbf{u}, \backslash}$ on language $\mathcal{M L}_{n}^{u,-}$ as:

$$
\gamma_{1} \Rightarrow_{c, \Gamma}^{c o} \gamma_{2}:=[c] \Gamma \wedge[-c] \neg \Gamma \wedge \neg[u]\left(\gamma_{1} \rightarrow \gamma_{2}\right)
$$

with $\gamma_{1}$ and $\gamma_{2}$ objective formulae.

A detailed comment of the definition is in order. The definition implements in modal logic the intuition summarized in Remark 1 and 2, and formalized in Definition 2: constitutive counts-as correspond to those classifications which are stated by the definition $\Gamma$ of the context $c$. Of course, for a given context, we can have a number of different equivalent definitions. The linguistic aspect comes thus into play and that is why $\Rightarrow_{c, \Gamma}^{c o}$ statements need to be indexed also with the chosen definition of the context, i.e. with the set of explicit promulgations of the normative system at issue. The warning "no logic of norms without attention to a system of which they form part" ( [16], pag. 29) is therefore taken seriously. As 
a result, constitutive counts-as statements can also be viewed as forms of speech acts creating a context: given that $\gamma_{1} \rightarrow \gamma_{2}$ is a formula of $\Gamma, \gamma_{1} \Rightarrow_{c, \Gamma}^{c o} \gamma_{2}$ could be read as "let it be that $\gamma_{1} \rightarrow \gamma_{2}$ with all the statements of $\Gamma$ and only of $\Gamma$ or, using the terminology of [21], "fiat $\Gamma$ and only $\Gamma$ ".

On the other hand, notice that because of this linguistic component of Definition 3 there is no logic, in a classical sense, of constitutive statements pertaining to one unique context description. That is to say, given a set of $\Rightarrow_{c, \Gamma}^{c o}$ statements, nothing can be inferred about $\Rightarrow_{c, \Gamma}^{c o}$ statements which are not already contained in the set $\Gamma$. How awkward this might sound it is perfectly aligned with the intuitions on the notion of constitution which backed Definition 3: constitutive counts-as are those classifications which are explicitly stated in the specification of the normative system. In a sense, constitutive statements are just given, and that is it. This does not mean, however, that constitutive statements cannot be used to perform reasoning. The following proposition touches upon the logical link relating constitutive statements pertaining to different but equivalent specifications of the same context.

Proposition 2. (Equivalent sets of $\Rightarrow_{c, \Gamma}^{c o}$ )

Let $\mathcal{M}$ be a $\mathrm{CXT}^{\top}$ model and $\Gamma$ and $\Gamma^{\prime}$ be context definitions containing no $\phi$ s.a. $\mathcal{M}=\phi$. Let $\Gamma$ contain $\left(\gamma_{1} \rightarrow \gamma_{2}\right) \wedge\left(\gamma_{3} \rightarrow \gamma_{4}\right)$, and let $\Gamma^{\prime}$ be obtained by $\Gamma$ substituting $\left(\gamma_{1} \rightarrow \gamma_{2}\right) \wedge\left(\gamma_{3} \rightarrow \gamma_{4}\right)$ with a formula $\left(\gamma_{5} \rightarrow \gamma_{6}\right)$. Then, it is valid that:

$$
\mathcal{M} \models[u]\left(\Gamma \leftrightarrow \Gamma^{\prime}\right) \rightarrow\left(\left(\gamma_{1} \Rightarrow_{c, \Gamma}^{c o} \gamma_{2} \wedge \gamma_{3} \Rightarrow_{c, \Gamma}^{c o} \gamma_{4}\right) \leftrightarrow\left(\gamma_{5} \Rightarrow_{c, \Gamma^{\prime}}^{c o} \gamma_{6}\right)\right)
$$

This states nothing but that the substitutions of equivalents in the description $\Gamma$ of a context always yields a set of constitutive $\Rightarrow_{c, \Gamma^{\prime}}^{c o}$ statements which is equivalent with the first set, provided that the the two definitions do not contain globally true statements.

Proof. Given that $[u]\left(\Gamma \leftrightarrow \Gamma^{\prime}\right)$ and the conditions on $\Gamma$ and $\Gamma^{\prime}$, it follows from the construction of $\Gamma^{\prime}$ that $[u]\left(\left(\left(\gamma_{1} \rightarrow \gamma_{2}\right) \wedge\left(\gamma_{3} \rightarrow \gamma_{4}\right)\right) \leftrightarrow\left(\gamma_{5} \rightarrow \gamma_{6}\right)\right)$.

Precise logical relations hold also between the two characterizations of Definition 1 and 3 and are investigated in the next section.

\subsection{Classification VS Constitution}

Since $\mathbf{C x t}^{\mathbf{u}} \backslash \backslash$ is an extension of $\mathbf{K} \mathbf{4 5} \mathbf{5}_{\mathbf{n}}^{\mathbf{i j}}$ it can be used to represent and reason about, at the same time, both $\Rightarrow_{c}^{c l}$ and $\Rightarrow_{c, \Gamma}^{c o}$ formulae. The logical relations between the two are formally explicated and studied in the following three propositions.

Proposition 3. $\left(\Rightarrow_{c}^{c l}\right.$ vs $\left.\Rightarrow_{c, \Gamma}^{c o}\right)$

In logic $\mathbf{C x t}^{\mathbf{u}, \backslash}$, the following formula is valid for every context definition $\Gamma$ containing $\gamma_{1} \rightarrow \gamma_{2}$ :

$$
\left(\gamma_{1} \Rightarrow_{c, \Gamma}^{c o} \gamma_{2}\right) \rightarrow\left(\gamma_{1} \Rightarrow_{c}^{c l} \gamma_{2}\right)
$$


Proof. It follows from Definitions 1 and 3: given that $\gamma_{1} \rightarrow \gamma_{2}$ is in $\Gamma$, then $\gamma_{1} \Rightarrow_{c, \Gamma}^{c o} \gamma_{2}$ implies that $[c] \Gamma$ holds. From which it follows that $[c]\left(\gamma_{1} \rightarrow \gamma_{2}\right)$.

The proposition translates the following intuitive fact: the statement of a constitutive rule guarantees, to say it with [14], the possibility of applying specific classificatory rules. If it is a rule of $\Gamma$ that self-propelled conveyances count as vehicles (constitutive sense) then self-propelled conveyances count as vehicles in the context $c$ defined by $\Gamma$ (classificatory sense).

Furthermore, it is noteworthy that formula 13 has a striking resemblance with the syntactic constraint that in [14] was imposed on the conditional minimal model semantics of counts-as conditionals: $A \Rightarrow_{c} B \rightarrow D_{c}(A \rightarrow B)$. This constraint was used to relate the logic of counts-as and the multi-modal $\mathbf{K D}_{\mathbf{n}}$ system chosen there as a logic of "general institutional constraints". Such a constraint, though intuitively motivated from a syntactic point of view, appears in that work a bit ad hoc since the choice for the $\mathbf{K D}_{\mathbf{n}}$ logic is not thoroughly investigated and it is even explicitly considered to be a "provisional proposal" ( [14], p.437). In our characterization, instead, the constraint emerges as a validity of the system, finding thus in our semantics a strong grounding and a clear formal motivation. It seems then safe to say that what authors in [14] meant under the label "constraint operative in institution c" was nothing but the notion of truth in a context (defined by a given normative system), i.e., what we have here represented via the $[i]$ operator. As a consequence, their strict implication under a $\mathbf{K D}_{\mathbf{n}}$ logic $\left(D_{c}\left(\gamma_{1} \rightarrow \gamma_{2}\right)\right)$ was in our view an attempt to capture the kind of statements that we investigated in [11] and that we have here called classificatory counts-as statements $\left(\gamma_{1} \Rightarrow_{c}^{c l} \gamma_{2}\right)$. A more detailed comparison of the two approaches can be found in [12].

The following result about constitutive counts-as statements also follows.

Proposition 4. (Impossibility of $\Rightarrow_{u, \Gamma}^{c o}$ )

Constitutive counts-as are impossible in the universal context $u$. In fact, the following formula is valid for every $\Gamma$ containing $\gamma_{1} \rightarrow \gamma_{2}$ :

$$
\left(\gamma_{1} \Rightarrow_{u, \Gamma}^{c o} \gamma_{2}\right) \rightarrow \perp
$$

Proof. The proposition is proved considering Definition 3: if $\gamma_{1} \Rightarrow_{u, \Gamma}^{c o} \gamma_{2}$ then $[u]\left(\gamma_{1} \rightarrow \gamma_{2}\right)$ and $\neg[u]\left(\gamma_{1} \rightarrow \gamma_{2}\right)$, from which contradiction follows.

In other words, what holds in general is never a product of constitution. This is indeed a very intuitive property strictly related to what discussed about Remark 1 in Section 2. As a matter of fact, that apples are classified as fruits is not due to any constitutive activity. Asserting that "apple always count as fruits" can only be true in a classificatory sense, while intending it as "apple always constitute fruits" is, logically speaking, nonsense. On the contrary, contextual classification statements are perfectly sound with respect to the universal context since no contradiction follows from sentences such as $\gamma_{1} \Rightarrow_{u}^{c l} \gamma_{2}$. This is related with the last property we report in this paper:

Proposition 5. (From $\Rightarrow_{c, \Gamma}^{c o}$ to $\Rightarrow_{c}^{c l}$ via $\Rightarrow_{u}^{c l}$ )

The following formula is valid for every $\Gamma$ containing $\gamma_{1} \rightarrow \gamma_{2}$ :

$$
\left(\gamma_{2} \Rightarrow_{c, \Gamma}^{c o} \gamma_{3}\right) \rightarrow\left(\left(\gamma_{1} \Rightarrow_{u}^{c l} \gamma_{2}\right) \rightarrow\left(\gamma_{1} \Rightarrow_{c}^{c l} \gamma_{3}\right)\right)
$$


Proof. Straightforward from Definition 1, Definition 3, Proposition 3 and the transitivity of classificatory counts-as (Proposition 1).

Formula 15 represents nothing but the form of the reasoning pattern occurring in the illustrative example with which our informal analysis started in Section 2: if it is a rule of $\Gamma$ that $\gamma_{2} \rightarrow \gamma_{3}$ ("self-propelled conveyances count as vehicles") and it is always the case that $\gamma_{1} \rightarrow \gamma_{2}$ ("cars count as self-propelled conveyances"), then $\gamma_{1} \rightarrow \gamma_{3}$ ("cars count as vehicles") in the context $c$ defined by normative system $\Gamma$. What is remarkable about this property is that it neatly shows how the two senses of counts-as both play a role in the kind of reasoning we perform with constitutive rules: the constitutive sense, though enjoying extremely poor logical properties, enables all the rich reasoning patterns proper of classificatory reasoning.

\subsection{Counts-as and the "transfer problem"}

The two meanings of counts-as behave differently with respect to the so-called transfer problem ( [14]). This problem can be exemplified as follows: suppose that somebody brings it about that a priest effectuates a marriage. Does this count as the creation of a state of marriage? In other words, is the possibility to constitute a marriage transferable to anybody who brings it about that the priest effectuates the ceremony?

From the standpoint of classificatory counts-as, the transfer problem is a feature of the formalism because $\Rightarrow{ }_{c}^{c l}$ enjoys the strengthening of the antecedent (Proposition 1). This is nevertheless what we would intuitively expect when interpreting counts-as statements as contextual classifications: whatever situation in which a priest performs a marriage ceremony, and therefore also the situation in which the priest is, for instance, forced to carry out the ritual, is classified as a situation in which a marriage state comes to be, with respect to the clerical statutes.

This is not the case for the constitutive reading of counts-as statements. In this view, counts-as statements represent the rules specifying a normative system. So, all that it is explicitly stated by the'institution of marriage' is that if the priest performs the ceremony then the couple is married, while no rule belongs to that normative systems which states that the action of a third party bringing it about that the priest performs the ceremony also counts as a marriage. Our formalization fully captures this feature. Let the 'marriage institution' $c$ be represented by the set of rules $\Gamma=\{p \rightarrow m\}$, i.e., by the rule "if the priest performs the ceremony, then the couple is married". Let then $t$ represent the fact that a third party brings it about that $p$. For Definition 3 the counts-as $(t \wedge p) \Rightarrow_{c, \Gamma}^{c o} m$ is just an undefined expression, because $((t \wedge p) \rightarrow m) \notin \Gamma$, that is, because the 'marriage institution' does not state such a classification.

\section{Discussion}

The main difference between our analysis and the formal approaches available -to our knowledge- in the literature at this moment $([1,6,14])$ is of a method- 
ological nature. In fact, though using modal logic like also [6,14], we did not proceed to the isolation of syntactic constraints that seem intuitive on the basis of the common usage of the term "counts-as". Instead, we made some precise interpretations formal of what counts-as statements mean (see Remarks 1-3), following hints from related disciplines such as legal and social theory. We deem the core advantage of this methodology to reside in the fact that the obtained formal characterizations could not be the result of mixing under the the same logical representation (in this case a conditional $\Rightarrow_{c}$ ) different semantic flavors that, from an analytical point of view, should be separated. For instance, suppose to establish whether transitivity is a meaningful property for a conditional characterization of counts-as. Tackling this issue syntactically amounts to check, on the basis of the natural usage of the term, the acceptability of the inference: if " $A$ counts as $B$ " and " $B$ counts as $C$ " then " $A$ counts as $C$ ". However, it can be case that the sense we naturally attach to the term "counts as" in the first premise is not the same sense we attach to the term "counts as" in the second premise or in the conclusion. This is indeed the case in the example with which our analysis started in Section 2 and which has been formalized in Proposition 5. A syntax-driven approach runs the risk of overlooking these ambiguities risking to import them in the formalization.

A last aspect worth mentioning concerns how our semantic analysis relates to syntax-driven approaches to counts-as which emphasize the defeasibility aspects of counts-as statements such as in particular [6]. Approaches analyzing counts-as as a form of defeasible rule or defeasible conditional deal in fact with something which is in essence different from what has been investigated here. In a sense they investigate yet another possible meaning of counts-as statements: "normally (or, unless stated otherwise), A counts as B in context C". Our approach stands to those ones as the classical approach to the formalization of a statement such as "all birds can fly" (bird $\subseteq$ fly) stands to those approaches formalizing it as a defeasible conditional or rule, that is, as "normally, all birds can fly".

\section{Conclusions}

The work has aimed at providing a clarification of the different meanings that can be given to counts-as statements. It has shown that there exist at least two senses in which 'counts-as can be said': the classificatory and the constitutive ones (a third one is investigated in [12]), both of which find grounds in legal and social theory literature. The modal logic characterizations of these two senses has made explicit the formal properties yielded by the two semantic options and, more noticeably, the logical relations holding between them.

\section{Acknowledgments}

We would like to thank the anonymous reviewers of DEON'06 for their helpful comments, and Prof. Andrew Jones for the inspiring discussions that motivated the research presented in this work. 


\section{References}

1. G. Boella and L. van der Torre. Attributing mental attitudes to normative systems. In Proceedings of AAMAS '03, pages 942-943, New York, NY, USA, 2003. ACM Press.

2. E. Bulygin. On norms of competence. Law and Philosophy 11, pages 201-216, 1992.

3. S. Buvač, S. V. Buvač, and I. A. Mason. The semantics of propositional contexts. Proceedings of the 8th ISMIS. LNAI-869, pages 468-477, 1994.

4. S. V. Buvač and I. A. Mason. Propositional logic of context. Proceedings AAAI'93, pages 412-419, 1993.

5. D.M. Gabbay, A. Kurucz, F. Wolter, and M. Zakharyaschev. Many-dimensional modal logics. Theory and applications. Elsevier, 2003.

6. J. Gelati, A. Rotolo, G. Sartor, and G. Governatori. Normative autonomy and normative co-ordination: Declarative power, representation, and mandate. Artificial Intelligence and Law, 12(1-2):53-81, 2004.

7. C. Ghidini and F. Giunchiglia. Local models semantics, or contextual reasoning = locality + compatibility. Artificial Intelligence, 127(2):221-259, 2001.

8. D. Grossi, H. Aldewereld, J. Vázquez-Salceda, and F. Dignum. Ontological aspects of the implementation of norms in agent-based electronic institutions. In Proceedings of NorMAS'05., pages 104-116, Hatfield, England, April 2005. AISB.

9. D. Grossi, F. Dignum, and J-J. Ch. Meyer. Contextual taxonomies. In J. Leite and P. Toroni, editors, Post-proceedings of CLIMA V, LNAI 3487, pages 33-51. Springer-Verlag, 2005.

10. D. Grossi, F. Dignum, and J-J. Ch. Meyer. Contextual terminologies. In F. Toni and P. Torroni, editors, Post-proceedings of CLIMA VI, LNAI 3900, pages 284-302. Springer-Verlag, 2006.

11. D. Grossi, J-J. Ch. Meyer, and F. Dignum. Modal logic investigations in the semantics of counts-as. In Proceedings of the Tenth International Conference on Artificial Intelligence and Law (ICAIL'05), pages 1-9. ACM, June 2005.

12. D. Grossi, J-J. Ch. Meyer, and F. Dignum. Classificatory aspects of counts-as: An analysis in modal logic. Under submission, 2006.

13. A. J. I. Jones and M. Sergot. Deontic logic in the representation of law: towards a methodology. Artificial Intelligence and Law 1, 1992.

14. A. J. I. Jones and M. Sergot. A formal characterization of institutionalised power. Journal of the IGPL, 3:427-443, 1996.

15. H. J. Levesque. All i know: A study in autoepistemic logic. Artificial Intelligence, (42):263-309, 1990 .

16. D. Makinson. On a fundamental problem of deontic logic. In P. McNamara and H. Prakken, editors, Norms, Logics and Information Systems. New Studies in Deontic Logic and Computer Science, pages 29-53. IOS Press, Amsterdam, 1999.

17. J.-J. Ch. Meyer and W. van der Hoek. Epistemic Logic for AI and Computer Science, volume 41 of Cambridge Tracts in Theoretical Computer Science. Cambridge University Press, 1995.

18. A. Peczenik. On Law and Reason. Kluwer, Dordrecht, 1989.

19. J. Searle. Speech Acts. Cambridge University Press, Cambridge, 1986.

20. J. Searle. The Construction of Social Reality. Free Press, 1995.

21. B. Smith. Fiat objects. Topoi, 2(20):131-148, 2001.

22. R. Stalnaker. On the representation of context. In Journal of Logic, Language, and Information, volume 7, pages 3-19. Kluwer, 1998. 